\title{
Does the efficacy of BCG decrease with time since vaccination? A meta analyis
}

Estimates of the protective efficacy of BCG vaccination (BCG PE) against tuberculosis from different studies range from negative to over $90 \%$. These estimates are heterogenous to a statistically significant degree. This study investigates the hypothesis that BCG PE decreases with time since vaccination, by performing a meta-analisys of the change in BCG-PE against tuberculosis over time observed in all nine randomised controlled trials which presented data separately for discrete time periods. For each trial, we derived log rate ratios for annual change in the rate of TB in

\section{London School of Hygiene and Tropical Medicine}

controls and the annual change in BCG PE. We also compared BCG PE in the first two years, and the last 10 years, to that in the rest of the trial, There was statistically significant heterogeneity between trials in the annual change in BCG PE. In six trials BCG $\mathrm{PE}$ decreased over time (in one of the trials this decrease was statistically significant) while in three of the trials it increased. The annual change in PE was not related to overall PE. Because of the heterogeneity, it would not be appropriate to calculate an overall trend in BCG PE with time.

\section{Trials reviewed}

\begin{tabular}{|c|c|c|c|c|}
\hline Location & Study - acumulation & Start date & $\begin{array}{r}\text { Maximum Years } \\
\text { of Follow-up }\end{array}$ & References \\
\hline \multicolumn{5}{|l|}{ Canada } \\
\hline Native Americans (USA) & 3008 American Indians Individuals & 1935 & 11 & $10-14$ \\
\hline Chicago (USA) & $3381 ?$ & 1937 & 23 & 15 \\
\hline Georgla (USA) & 4839 children & 1949 & 20 & 16,17 \\
\hline Puerto Rico & 77927 children & 1949 & 20 & 18,19 \\
\hline Georgia (USA) & 34567 individuals of $5+$ years & 1950 & 20 & $18,20 \cdot 23$ \\
\hline England & 26465 adolescents and early adults & 1950 & 20 & $7,24-27$ \\
\hline Madanapalle (S. India) & 10872 nigers & 1950 & 21 & $28-30$ \\
\hline Madras (S. India) & 90000 gers & 1968 & 15 & 31,32 \\
\hline
\end{tabular}

Trials reviewed

\begin{tabular}{|c|c|c|c|c|c|}
\hline \multicolumn{3}{|c|}{ Summary protection in trials: } & \multicolumn{3}{|c|}{ Rate ratios $(95 \% \mathrm{CI})$ for change in effect of BCG over time } \\
\hline Trial & $\mathrm{PE}(95 \% \mathrm{Cl})$ & $\begin{array}{l}\text { RR }(95 \% \mathrm{Cl}) \\
\text { for annual change } \\
\text { in rate of TB } \\
\text { in controls }\end{array}$ & $\begin{array}{l}\text { Annual change } \\
\text { in effect of vaccine }\end{array}$ & $\begin{array}{l}\text { Effect after the first } \\
2 \text { years, compared } \\
\text { to the first } 2 \text { years } \\
\text { of the trial }\end{array}$ & $\begin{array}{l}\text { Effect after the first } \\
10 \text { years, compared } \\
\text { to the first } 10 \text { years } \\
\text { of the trial }\end{array}$ \\
\hline $\begin{array}{l}\text { Saskatchewan } \\
\text { (Canada) }\end{array}$ & $78.5(46.6,92.8)$ & $0.969(0.866 .1 .086)$ & $\begin{array}{l}0.949 \\
(0730,1,232)\end{array}$ & $1.841(0.268,12.649)$ & - \\
\hline $\begin{array}{l}\text { Native Americans } \\
\text { (USA) }\end{array}$ & $80.8(73.6,86.2)$ & $0.968(0.919,1.019)$ & $\begin{array}{l}1.182 \\
(1.036,1.348)\end{array}$ & $3.449(1.807,6.582)$ & - \\
\hline $\begin{array}{l}\text { Chicago } \\
\text { (USA) }\end{array}$ & $72.5(51.6,85.3)$ & $0.780(0.713,0.855)$ & $\begin{array}{l}0.906 \\
(0.761,1.078)\end{array}$ & $0.336(0.069,1.641)$ & $0.522(0.044,6.157)$ \\
\hline $\begin{array}{l}\text { Georgia School } \\
\text { (USA) }\end{array}$ & $-56.3(-905.0,69.6)$ & $1.026(0.838,1.257)$ & $\begin{array}{l}0.928 \\
(0.717,1.201)\end{array}$ & - : & $0.333(0.017,6.648)$ \\
\hline $\begin{array}{l}\text { Puerto Rico } \\
\text { (USA) }\end{array}$ & $27.9(9.6,42.4)$ & $0.940(0.910,0.970)$ & $\begin{array}{l}1,007 \\
(0.965,1.050)\end{array}$ & $0.859(0.469,1.572)$ & $1.208(0.768,1.899)$ \\
\hline $\begin{array}{l}\text { Georgia } \\
\text { Community } \\
\text { (USA) }\end{array}$ & $6.2(-55.5,43.6)$ & $0.973(0.915,1.034)$ & $\begin{array}{l}0.952 \\
(0.872,1.038)\end{array}$ & ln: & $0.810(0.310,2.114)$ \\
\hline MRC & $76.7(69.2,82,6)$ & $0.826(0.801,0.851)$ & 0.919 & $0.783(0.406,1.510)$ & $0,359(0.169,0.785)$ \\
\hline $\begin{array}{l}\text { (England) } \\
\text { Madanapalle } \\
\text { (India) }\end{array}$ & $19-4(-28,4,50.0)$ & $1.106(1.051,1.165)$ & $\begin{array}{l}(0.868,0.973) \\
0.933 \\
(0.857,1.016)\end{array}$ & : & $0.163(0.034,0.782)$ \\
\hline $\begin{array}{l}\text { Madras } \\
\text { (India) }\end{array}$ & $-2.9(-24.0,14.4)$ & $1.133(1.08,80)$ & $\begin{array}{l}1.044 \\
(0.995,1.095)\end{array}$ & $2.102(0.846,5.222)$ & $1.031(0.717,1.482)$ \\
\hline $\begin{array}{l}\mathrm{x}^{2} \text { test for } \\
\text { heterogeneity }\end{array}$ & & & $\begin{array}{l}22.85(9 \mathrm{df}) \\
p=0.004\end{array}$ & $\begin{array}{l}16.82(6 \mathrm{df}) \\
p=0.005\end{array}$ & $\begin{array}{l}13.05(7 \mathrm{df}) \\
p=0.042\end{array}$ \\
\hline
\end{tabular}

